\title{
EFFICIENT DDOS ATTACKS SECURITY SCHEME USING ASVS
}

\author{
Vikneshkumar. $D^{1}$, Srinivasan. ${ }^{2}$, Nagulanand. $M^{3}$ \\ ${ }^{1,2}$ Assistant professor, ${ }^{3} P G$ scholar, Department of computer science, SriGuru Institute of technology, Tamilnadu, India, \\ vikneshkumard@gmail.com,srinivasanl1982@gmail.com,msnagul07cs48@gmail.com
}

\begin{abstract}
A distributed Denial of Service (DDoS) attack enables higher threats to the internet. There are so many scheme designed to identify the node which is to be attacker node. The real process is such as we want to trace the source of the attacker and enable security to our network. The protocol introduced here, called Adaptive Selective Verification with Stub (ASVS) is shown to use bandwidth efficiently and uses stub creation. The Stub procedure to reduce the server load at the time of emergency and congestion. Using this stub idea we can store the ASVS protocol procedure in the server and we can have the stub in the every client so that we can detect the hacker system by the client itself. We use omniscient protocol which enables to send information about the attacker to all the clients.
\end{abstract}

Keywordss: Adaptive Selective Verification With Stub (ASVS), Distributive Denial Of Service Attacks (DDoS) Flooding, Performance Analysis.

\section{INTRODUCTION}

DDoS stands for Distributed Denial of Service attack. It is a form of attacks where a lot of infected computers which are under the control of the attacker are used to either directly or indirectly to flood the targeted systems as a victim, with a huge amount of information and block in order to prevent original users from accessing them (mostly web servers which host websites).They can occur at link, network, transport, or application layers.

They can be sudden and dramatic or gradual and re strained. Intention of these attacks are aimed at disabling services, are easy to confuse between original user and hacker. Most of the networks are failed to design with DDoS thus they meet the vulnerable effects. This rich collection of attack vectors combines with various options for which are changed to affect a countermeasure. The time required to process these requests were of high range which degrades the service to available clients and make the Cost over by the service provider for provisioning.

There are various scheme for this problem namely currency based mechanism, which enables a server under attack enables to use all form of resources from clients thus it increases the process of server. This mechanism enables to assume bandwidth as currency and enables to send dummy bytes for connection configuration. These dummy bytes enable to congestion in network and enables traffic.

In this paper we introduce Adaptive Selective Verification with Stub(ASVS), which is a distributed adaptive[1] scheme for protecting attackers efforts from deny service to true clients based on selective verification and enables perform operation using stub in each client procedure. Our scheme uses bandwidth efficiently by adapting independent such that each client will have bandwidth allocation based on distance the client is located. If hacker node is been found means it automatically cancels bandwidth of the respective hacked client node .Stub libraries must be installed on client and server side, such that it enables information pass through every client and server. we evaluate its performance as compared to an "omniscient" protocol in which all attack parameters are instantaneously made known to all clients as well as the servers. We enable to show that ASVS closely approximates the performance of this omniscient protocol and enables guaranteed performance

In Fig. 1, shows the simple architecture of Distributed Denial of Service (DDoS) attack model.

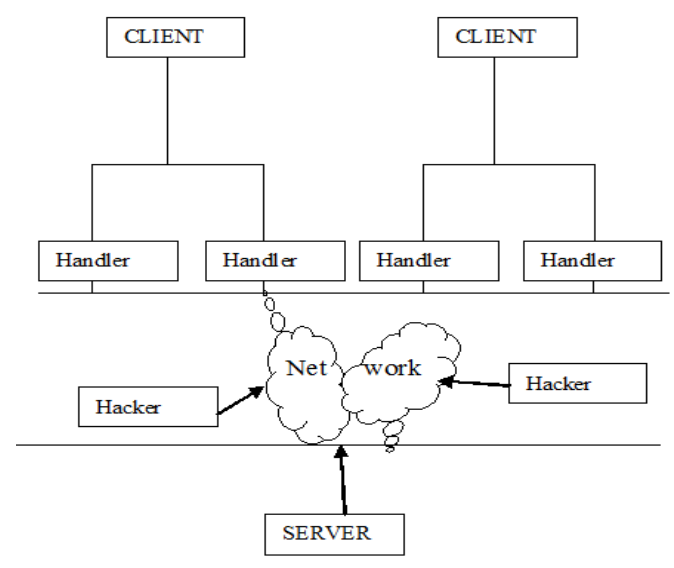

Fig 1: Distributed Denial of Service (DDoS) attacks 


\section{RELATED WORKS:}

The related work enables the ways in which various information we get for our scheme such that A. Yaar[7] shows that Critical infrastructures and businesses alike flash crowds which are suppose to be vulnerable for DoS attacks .SIFF, a Stateless Internet Flow Filter, which is an end host selectivity scheme which stops the flow of individual to reach its network. The division of all network traffic into two classes privileged which enables control of recipient control prioritized packets and unprivileged. Privileged channels are established through a handshake exchange capability. D. K. Y. Yau[8] shows that strangle which can be the leaky-bucket rate at which a router can forward designed packets for the server. Hence, aggressive packets are converged engulf the server, thus it routers participating proactively regulate the contributing packet rates to more moderate levels, thus prevention an imminent attack. In allocating the server capacity among the routers, a notion of level- max-min fairness is evaluated to represent of a control-theoretic model under a variety of system parameters.

\section{RESEARCH METHODOLOGY}

Any system is able to be affected by DDoS attack, the objective of our scheme is to adapt an algorithm which can be helpful in tracing back the source of DDoS attacks.

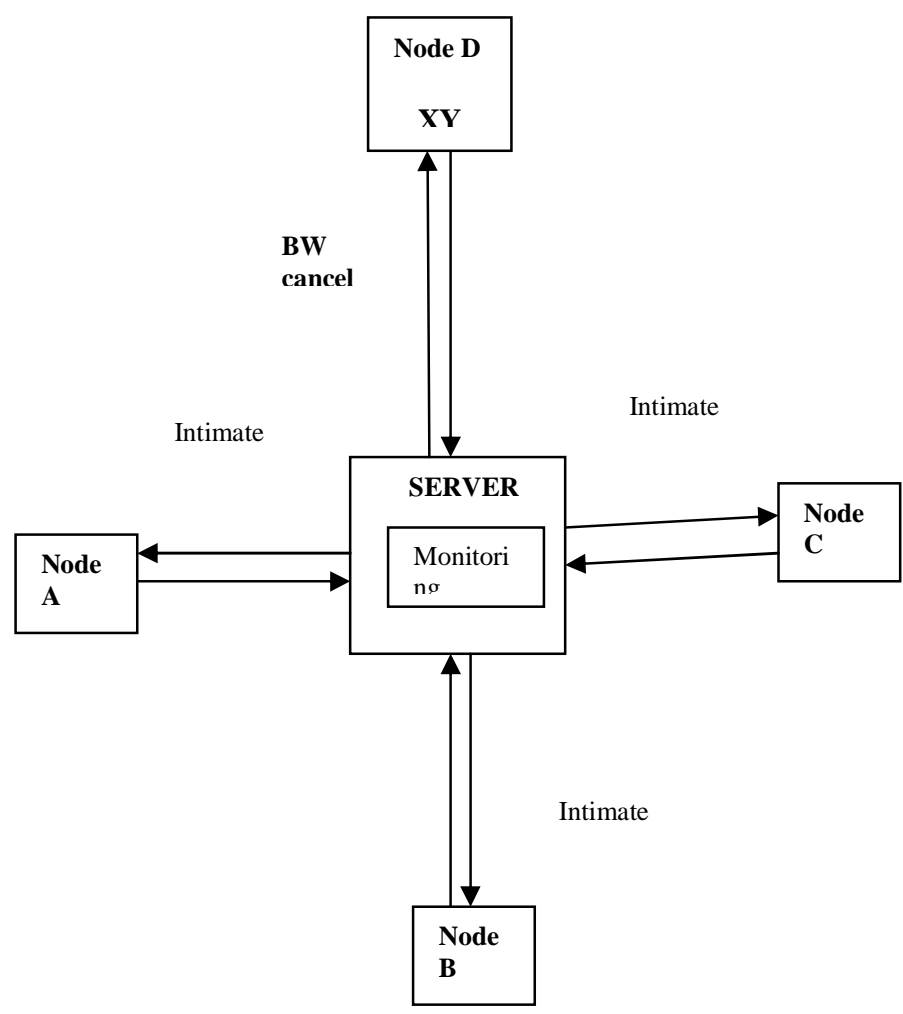

Fig2: Working of ASVS scheme
The figure represents the activities of server such that it monitors all the activities of client system. If any deviation or change of bandwidth of client system means it identifies as a hacker system and it does two processes. First, it cancels bandwidth of particular node and passes the information of hacker node to all nodes present in the network. The idea is to use secure information transformation between client and server.

\subsection{Shared Channel Model}

The intruders use handler machines to specify the attack type and the victim's address and wait for the appropriate moment in order to mount the attack. The agent node enables to send a stream of packets to the victim, thereby flooding the hacked system with dummy loads and draining its resources. In this way, the attackers cause to be the victim node which were unavailable to rightful clients and obtain unlimited access such that it causes damage to lawful system. The volume of traffic which is to be so high that the networks enables to ahv a low performance which connect the attacking machines to the victim.

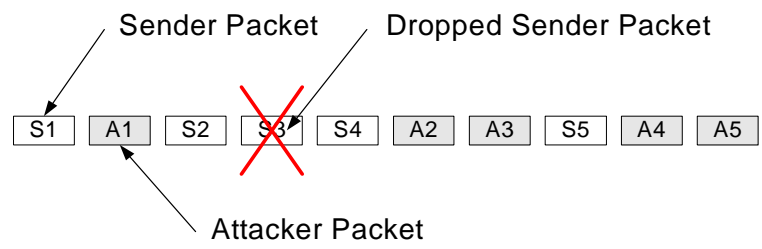

Fig 3: Shared Channel of Packets

The provision of services over the networks where no longer possible, such that these services are denied by clients. Thus, the network that has been held back by the attacker's load which are measured as another victim of the DDoS attack.

In keeping with theoretical review of the shared channel model that was expressed by the authors to model DoS attacks are assumption ,that the attack factors are uniformly bounded, for some fixed, though the upper bound on the attack factors which are considered to be very large.

Clearly, when, the attack overwhelms the server's capacity to process all requests unless there is a mechanism to efficiently handle the attack packets.

\subsection{Node Verification}

A system and method determines the proximity of the target node to the source node from the time required to communicate messages within the node-verification protocol. The node-verification protocol includes a query-response sequence, wherein the source node communicates a query to the target node, and the target node communicates a 
corresponding response to the source node. The target node is configured to communicate two responses to the query: a first response that is transmitted immediately upon receipt of the query, and a second response based on the contents of the query. The communication time is determined based on the time duration between the transmission of the query and receipt of the first response at the source node and the second response is compared for correspondence to the query, to verify the authenticity of the target node.

This strategy was introduced in the context of authenticated broadcast using selective verification and extended to general Internet protocols in using bandwidth selection. Selective verification enables the clients to send extra requests to the server such that the server samples from these requests randomly. In this mechanism, the attack enables to transfer the newly true system from the attacker. More clearly, the attack tools that are installed on the attacker system which includes a specific method for accepting a connection from the true system and sending a file to it that contains the attack tools. This back-channel file copy enables to check intruders by means of simple port listeners.

This technique is very effective in receding the effects of a DoS attack if a sufficient level of client back channel file is used in every client machine.

\subsection{Flooding attacks:}

Flood attacks are suppose to be happen at high rate such that it blocks the speed of server process as well as client. This is generated by creating high traffics from many machines, which may in number of thousands and distributed all over the world. Huge number of the flood packets from the attackers will devastate the target such that it degrades the performance. The high flood rate attacks are reviewed in with the UDP attacks and TCP attacks. These are pointed into as high rate flood attacks because these attacks are launched by flooding a massive amount of TCP or UDP data grams to overcome the victim Flood attacks. These enables to internet threat for monitors [5] which were of flooding-based Distributed Denial of Service (DDoS) attack is performed by the attacker by sending a huge amount of unwanted traffic to the victim system and it is the very commonly used attack by the attacker.

To launch a DdoS attack, malicious users builds a network of computers that which cause to produce the large volume of traffic needed to deny services to computer users. To establish this type of attack, attackers discover vulnerable sites which hosts on the network. The traffic rate is high and it must be adjusted in order to make them un detected by the traditional flood detector which regards high rate of incoming traffic as attack.
Thus we check our packet distribution through output such that if rate of data save is higher, then we notice that flooding has been occurred.

\subsection{Hop Sequence (Unidirectional)}

It is usable in unidirectional communications, requires no server state, and makes no assumptions about network congestion. However, extra client requests are a cost that should ideally be avoided when there is no attack and used proportionately to the strength of attack when there is one. The provoked capability approach to limit the effects of network denial-of-service attacks, and presented and evaluated (a revised version of) TVA[4], the complete and practical capability-based network architecture. As a complete system, it details the operation of capabilities along with protections for the initial request exchange, consideration of destination policies for authorizing senders, and ways to bound both router computation and state requirements. The evaluation of TVA using a combination of simulation, implementation, and analysis. When TVA is used, even substantial floods of legacy traffic, request traffic, and other authorized traffic have limited impact on the performance of legitimate users.

This can be done by placing inline packet processing boxes near legacy routers, with incremental deployment providing incremental gain. The aim of this scheme is to do this by surrendering unidirectional communication capabilities and developing an acknowledgment-based adaptive technique.

\subsection{Adaptive Selective verification (ASV)}

The protocol introduced here, called Adaptive Selective Verification (ASV)[1], is shown to use bandwidth efficiently and does not require any server state or assumptions about network congestion. The main results of this scheme are to formulate a optimal performance and a proof that ASV is best possible. Srivastsa et al. [2] this scheme enables to select the node appropriately such that it enables to identify the utility of clients. More the feedback got from the client enables to improve performance of selection, which enables to store this history to the server. Wang et al. [3] show how to provide adaptation for client puzzles. Because of the nature of the client puzzle schemes, where the cost factor of the defense on the server is minimal, their proposal mainly focuses on cost minimization for the clients.

A stub in distributed computing is a piece of code used for converting parameter which were passed during a Remote Procedure Call (RPC).The main design of an RPC is to allow a local computer (client) to call remote procedures on a remote computer (server). The client and server use different address, so conversion of parameters in a function call is used and it is performed, if not the values of those parameters cannot be used, because of pointers to pointing to different location in computer memory. The client and server use different data 
representations for simple parameters. Stubs are used to perform the parameter conversion, so a Remote Function Call resembles like a local function call on the remote computer. Stub libraries must be used on client and server side such that client stub is responsible for conversion of parameters used in a function call and de conversion after server execution. A server skeleton is to be the stub on server side, is responsible for de conversion of parameters which passed by the client and conversion as a results of execution of the function.

\section{CONCLUSIONS}

In this paper we proposed effective and efficient scheme which enables to identify and select hacker node adaptively such that these identification can be done by client without interventions of server node. The stub creation involves the client information such that these are passed to every client and server when possible. Thus it provides reduce the burden of server by checking the hacker node. In addition to that, this scheme enables traffic on internet cross is minimal when it is compared to non adaptive counterpart.

\section{REFERENCES}

[1]Adaptive Selective Verification: An Efficient Adaptive Counter measure to Thwart DoS Attacks Sanjeev Khanna, Santosh S. Venkatesh, Member, IEEE, Omid Fatemieh, Fariba Khan, and Carl A. Gunter, Senior Member, IEEE, Member, ACM, IEEE/ACM transactions on networking, vol. 20, no. 3, june 2012.

[2]M. Srivatsa, A. Iyengar, J. Yin, and L. Liu, "a middleware system for protecting against application level denial of service attacks," in Proc. Middleware, 2006, pp. 260-280.

[3]X. Wang and M. K. Reiter, "Defending against denial-ofservice attacks with puzzle auctions," in Proc. IEEE Symp. Security Privacy, Washington, DC, 2003, pp. 78-92.

[4]X. Yang, D. Wetherall, and T. Anderson, "TVA: A DoSlimiting network architecture," IEEE/ACM Trans. Netw., vol. 16, no. 6, pp. 1267-1280, Dec. 2008.

[5]Flooding attacks to internet threat monitors(Itm): modeling and counter measures using botnet and honey pots, k.munivara prasad and a.rama mohan reddy, m.ganesh karthik International Journal Of Computer Science \& Information Technology (Ijcsit) Vol 3, No 6, Dec 2011

[6]Geographical Division Traceback for Distributed Denial of Service, Viswanathan, A., V.P. Arunachalam and S. Karthik, Journal of Computer Science 8 (2): 216-221, 2012 ISSN 15493636

[7] A. Yaar, A. Perrig, and D. X. Song, "SIFF: A stateless internet flow filter to mitigate DDoS flooding attacks," in Proc. IEEE Symp. Security Privacy, 2004, pp. 130-143.

[8] D. K. Y. Yau, J. C. S. Lui, F. Liang, and Y. Yam, "Defending against distributed denial-of-service attacks with max-min fair server-centric router throttles," IEEE/ACM Trans. Netw., vol. 13, no. 1, pp. 29-42, Feb. 2005.

\section{BIOGRAPHIES}

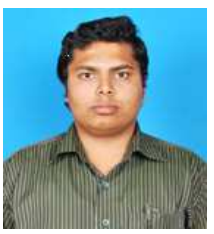

Viknesh Kumar. D has received B.Tech degree in Information Technology from Anna University Coimbatore, Tamilnadu, India. He received his M.E degree in Computer Science from Anna University, Chennai, Tamilnadu, India. He is working as Assistant Professor in SriGuru Institute of Technology, Coimbatore, Tamilnadu. His area of interest includes Data structures, Network security, and Software engineering.

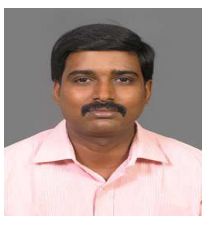

Srinivasan. L has received B.E. degree in Information Technology from Bharathiar University Coimbatore, Tamilnadu, India. He received his M.Tech degree in Information Technology from Anna University, Coimbatore, Tamilnadu, India. He is working as Assistant Professor in SriGuru Institute of Technology, Coimbatore, Tamilnadu. He is pursuing his Ph.D in Data mining in Anna University Chennai, Tamilnadu, India. His area of interest includes Data mining, Data structures, and System software.

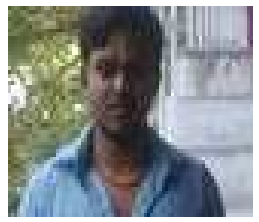

Nagulanand. M has received B.E degree in Computer Science from Anna University Chennai, Tamilnadu, India. He is doing his M.E degree in computer science SriGuru Institute of Technology, Coimbatore Tamilnadu, India. His area of interest includes Data structures, Networking, Data mining. 